\title{
Studying the Correlation between Axial Length and Retinal Nerve Fiber Layer and Macular Thickness by Spectral-Domain Optical Coherence Tomography \\ R.H.Abd AL-Fatah, A.H.El Habbak, O.M.Kamal and A.A.Shebl
}

Ophthalmology, Dept., Faculty of Medicine, Benha Univ., Benha, Egypt

E-mail:RadwaHossam82@gmail.com

\begin{abstract}
Background: The ability to see well is critical in one's life. Our retina is similar to a film in a camera, generating electrical impulses that are then sent to the brain, where they are interpreted as visual pictures by retinal nerve fibres in the brain. A healthy macula provides the sharpest vision, which is essential for jobs that need a lot of visual processing power. To ensure the efficacy of treating macular injuries, it is essential to measure the thickness of the macula before and after therapy. Diagnostic imaging using OCT offers in vivo study of various retinal layers and anterior segment with 1-15 m resolution without contacting or invasively implanting any equipment into the patient's body Because it accurately measures RNFL and macular thickness and identifies early structural changes, it becomes an essential diagnostic tool for glaucoma, macular edoema, and other retinal and optic nerve disorders. It may also be used to follow up on patients with macular degeneration. It is now possible to create 3D pictures with unprecedented speed and quality thanks to the advent of the new SD-OCT technology. RNFL and macular thickness may be evaluated in a safe, consistent, rapid, and objective manner using this approach. Studying pRNFL and macular thickness in hyperopia, myopia, and emmetropia using spectral domain optical coherence tomography has the goal of learning how they relate to acuity levels (AL). Methods: The 45 eyes of 23 healthy volunteers were sorted into three categories based on their axial length: Group I (myopic) consists of 15 people (axial length: 24.6-27 mm). Eyes in the second group (Emmetropic) have (axial length: 22.6-24.5 mm). There are 15 eyes in this group that are hypermetropic (axial length: 20-22.5 mm). Spectral-domain Optical Coherence Tomography was utilised to quantify macular thickness and peripapillary RNFL thickness in all quadrates after a complete clinical examination of the participants. Results: To minimise the impact of age-related retinal changes, we structured the present research to include participants ranging in age from 14 to 40. Age had no effect on macular or pRNFL thickness, which were found to be identical. Our research identified no significant differences in macular thickness and RNFL thickness across other demographic characteristics including gender and eye preference. Because of this, these aspects aren't relevant when trying to determine what RNFL readings are typical. The research groups did not vary in central macular thickness (CMT). In all quadrants (temporal, inferior, and nasal), except in the superior quadrant, parafoveal thickness does not vary significantly. However, there was a strong negative association between it and other variables across research groups. There was a strong negative connection in the superior, temporal, and nasal quadrants for perifoveal thickness, but there was no difference in the inferior quadrant. This suggests that All quadrants had a thicker parafoveal zone than the perifoveal region. The "double hump pattern" and the "ISNT rule" were evident in our analysis of pRNFL thickness. The AL demonstrated a negative relationship with the thickness of the RNFL. In both the superior and inferior quadrants, this was statistically significant. In terms of the temporal and nasal quadrants, there was no significant difference between the research groups. It can be concluded that the lengthening of AL has a significant negative correlation with the macula retinal thickness in the superior, nasal and temporal quadrants as well as the inner parafovea in the superior quadrant. However, there was no correlation between AL lengthening and the macula's central thickness. In all three groups, pRNFL exhibited the distinctive "double hump pattern" and adhered to the "ISNT rule." When it came to the temporal and nasal quadrants, there was no statistically significant difference between the research groups in terms of RNFL thickness and axial length. To accurately assess the thickness of various retinal tissues, such as RNFL, OCT is a helpful tool. This helps in early glaucoma diagnosis, follow-up, and treatment of patients to prevent them from irreversible vision loss.
\end{abstract}

Key words: Axial Length, Retinal Nerve Fiber Layer, Macular Thickness, Spectral, Domain, Optical Coherence Tomography.

\section{Introduction}

RGC axons are made up of axonal membranes, microtubules, neurofilaments, and mitochondria, and form the retinal nerve fibre. Another important part of the human visual system, it transports visual information from cones and rods to the brain through the optic nerve head, where it is processed by the brain (ONH) [1].

While in the circum-papillary regions (i.e., around the $\mathrm{ONH}$ ), nerve fibre bundle distribution is primarily circular, with striations of fibre bundles from the ONH's temporal part flowing into the foveal region where a cluster congregates at the foveal centre while others divert to more distant temporal positions. The fibre bundle striations are radial in the superior, nasal, and inferior directions and bend in the temporal direction, away from the ONH. [2].

The retinal nerve fibre layer (RNFL) is the retinal layer that houses the nerve fibre bundles. It is placed on the retina's surface, behind the ILM and ahead of the retinal ganglion cell layer (RGC). Healthy eyes have RNFL thicknesses ranging from around 10 microns (near the fovea) to 400 microns (at the $\mathrm{ONH}$ edge) [1]. 
With optical coherence tomography (OCT), the retina and optic nerve head may be seen in crosssection, making it possible to quantify the thickness of the retinal nerve fibre layer (RNFL) and the macula in a non-contact way. [3].

RNFL and macula measurements are now possible using a novel spectral domain OCT (SDOCT) with fast image collection, improved axial resolution $(15 \mathrm{~m})$, and creation of $3 \mathrm{D}$ pictures. The SDOCT technique also makes it possible to segment and quantify the different retinal layers with the use of computer systems. [4].

In comparison to emmetropes, the average thickness of the peripapillary RNFL was lower in myopic and thicker in hypermetropic eyes in the research by Oner et al. [5]. According to the findings of Sowmya et al. [3], RNFL thickness decreased with increasing myopia, but it rose with increasing hypermetropia.

As with histopathologic research, further studies using the third-generation Stratus OCT has shown a link between average macular thickness and refractive error and axial length in healthy participants. [6].

The impact of axial length on inner macular thickness is still up for debate. Some studies have established a link between axial length and the thickness of the inner macular slit, whereas others have not. An ultrasonic scan is the contact approach that needs a topical anaesthetic and prior keratometery to utilise. [7, 8].

Rather, the A-scan gadget measures the time it takes a sound wave to travel from the cornea to the retina in microseconds. To calculate the distance, you'll need to know how fast the sound is travelling and how far it has gone. [9].

This research used Spectral Domain Optical Coherence Tomography to look at the relationship between axial length, the thickness of the peripapillary retinal nerve fibre layer, and the thickness of the macula in myopic, hypermetropic, and emmetropic eyes.

\section{Patients and Methods}

\subsection{Study design}

This is a cross sectional study which was performed on 45 eyes of 25 subjects recruited from Ophthalmology Outpatient clinic of Benha University Hospitals.

On the basis of axial length, eyes were divided into three groups :

- Group I (Myopic): 15 eyes with (axial length: 24.6-27 mm).

- Group II (Emmetropic): 15 eyes with (axial length: 22.6-24.5 mm).

- Group III (Hypermetropic): 15 eyes with (axial length: $20-22.5 \mathrm{~mm}$ ).

\subsection{Study population:}

2.3. Inclusion criteria:

I-Subjects were included in this study with criteria of:

- Aged from 18-40 year, both males and females.

- Not diabetic, not hypertensive.

- Good candidates for OCT imaging, no media opacity ( in cornea, lens and vitreous).

- No posterior segment pathology.

- No history of glaucoma, retinopathy, retinal therapy, or other

- Intraocular surgical intervention.

\subsection{Exclusion criteria}

II-Study excluded following people with:

- Age below 18 or above $40 \mathrm{y}$.

- Subjects with media opacities or Anterior segment pathology.

- Previous history of ocular surgery or trauma.

- History of glaucoma, optic nerve or macular disease.

- Intraocular pressure (IOP) $>21 \mathrm{mmHg}$.

- Cup/Disc ratio $>0.5$ or asymmetry $>0.2$

\subsection{Methods}

All Subjects had full examination through:

History taking including:

- Age.

- Sex.

- History of glasses or any surgical refractive correction.

- Past Ocular history (disease, surgery).

- Any associated systemic disease.

Ocular examination:

Complete ophthalmological examination done, including:

- Pupillary reaction.

- Uncorrected visual acuity (UCVA) using Snellen's chart testing.

- Best corrected visual acuity (BCVA) using Snellen's chart testing after Autorefractometry.

- Anterior segment assessment by slit lamp examination.

- Intraocular pressure (IOP ) measurement.

- Fundus examination using Indirect Ophthalmoscope with +20 D lens (to evaluate the periphery of the retina) and Biomicroscopy with $+90 \mathrm{D}$ lens (for evaluating the posterior pole).

- Axial length measured by using (SONOMED Pac Scan Plus 300 A+, USA ) A-scan Ultrasound Biometry. After application of $0.4 \%$ Benoxinate Hydrochloride on the both eyes, applanation ultrasound probe directly touches the cornea estimating the axial length of the eye. 


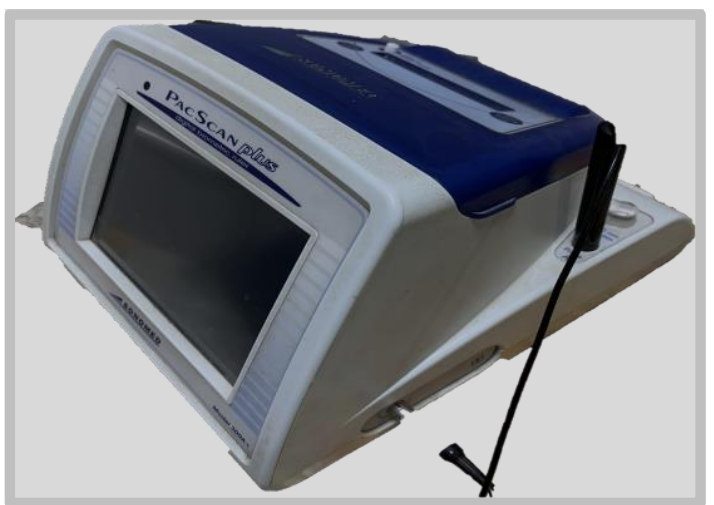

Fig. (1) A-scan Ultrasound Biometry.

- $\quad$ Spectral-Domain Optical Coherence Tomography (Structural Assessment) examination with pupils dilated in all subjects with $1 \%$ cyclopentolate. OCT done using 3D OCT 2000 FA (TOPCON CORPORATION, Tokyo, Japan). 3D macula mode and 5 lines cross scan mode used to obtain high quality images for assessment of Macular thickness and 3D cubic of $(6 \mathrm{mmX6mm})$ area used to assess Peripapillary RNFL thickness in all quadrates.

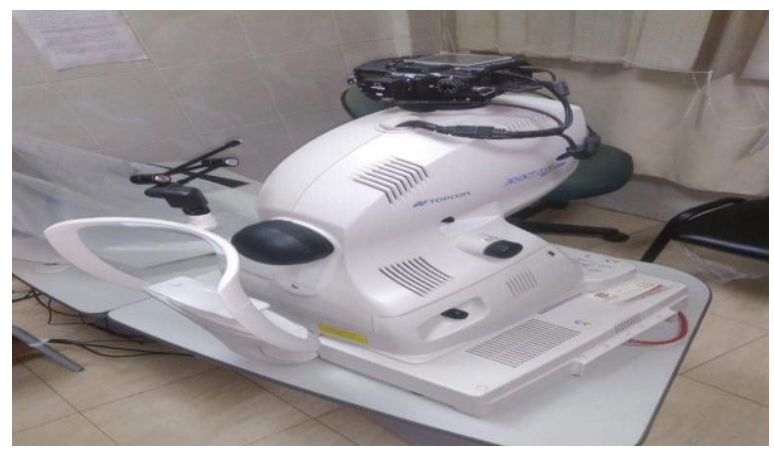

Fig.2: Spectral-Domain Optical Coherence Tomography (SD-OCT).

\subsection{Statistical methods}

Data management and statistical analysis were done using SPSS version 25. (IBM, Armonk, New York, United States). Quantitative data were assessed for normality using the Shapiro-Wilk test and direct data visualization methods. According to normality, numerical data were summarized as means and standard deviations or medians and ranges. Categorical data were summarized as numbers and percentages. Quantitative data were compared between study groups using one-way ANOVA or Kruskal Wallis test for normally and non-normally distributed numerical variables, respectively. Categorical data were compared using the Chi-square or Fisher's exact test, if appropriate. All statistical tests were two-sided. $\mathrm{P}$ values less than 0.05 were considered significant.

\section{Results}

\subsection{General characteristics}

$>$ BCVA showed an overall significant difference between the study groups $(\mathrm{P}$-value $=0.03)$. Posthoc analysis showed that it was significantly higher in group III (0.3) than group II (0.0).

$>$ No significant differences were observed regarding age $(\mathrm{P}$-value $=0.3760$, gender $(\mathrm{P}$-value $=0.871)$, side $(\mathrm{P}$-value $=0.929)$, and IOP $(\mathrm{P}$-value $=0.537)$. Table (1).

Table (1) General characteristics in the studied groups

\begin{tabular}{|c|c|c|c|c|c|}
\hline & & $\begin{array}{c}\text { Group I } \\
(\mathrm{n}=\mathbf{1 1} \mathrm{pts}, 15 \text { eyes })\end{array}$ & $\begin{array}{c}\text { Group II } \\
(\mathrm{n}=\mathbf{1 0} \text { pts, } 15 \text { eyes })\end{array}$ & $\begin{array}{c}\text { Group III } \\
(\mathrm{n}=8 \mathrm{pts}, 15 \mathrm{eyes})\end{array}$ & P-value \\
\hline Age (years) & Mean \pm SD & $31 \pm 9$ & $32 \pm 8$ & $26 \pm 9$ & 0.376 \\
\hline \multirow{2}{*}{ Gender } & Males $\quad \mathrm{n}(\%)$ & $3(27.3)$ & $2(20.0)$ & $3(37.5)$ & 0.871 \\
\hline & Females $\mathrm{n}(\%)$ & $8(72.7)$ & $8(80.0)$ & $5(62.5)$ & \\
\hline Side & $\begin{array}{ll}\text { OD } & \mathrm{n}(\%) \\
\text { OS } & \mathrm{n}(\%)\end{array}$ & $\begin{array}{l}9(60.0) \\
6(40.0)\end{array}$ & $\begin{array}{l}7(46.7) \\
8(53.3)\end{array}$ & $\begin{array}{l}8(53.3) \\
7(46.7)\end{array}$ & 0.929 \\
\hline $\begin{array}{l}\text { BCVA } \\
\text { (Logmar) }\end{array}$ & Median (range) & $0.18(0-1)^{\mathrm{a}, \mathrm{b}}$ & $0(0-0.78)^{a}$ & $0.3(0-1)^{\mathrm{b}}$ & 0.03 \\
\hline IOP & Mean \pm SD & $16 \pm 2$ & $16 \pm 1$ & $16 \pm 1$ & 0.537 \\
\hline
\end{tabular}


$>$ One-way ANOVA or Kruskal Wallis test was used for numerical data. Chi square or Fisher's exact test was used for categorical data

\subsection{Central macular thickness}

Central macular thickness (CMT) showed a nonsignificant difference between the study groups $(\mathrm{P}-$ value $=0.115)$. Table $(2)$.

\subsection{Parafoveal thickness}

$>$ Regarding parafoveal thickness, only the superior quadrant showed an overall significant difference between the study groups $(\mathrm{P}$-value $=0.013)$. Post hoc analysis showed that it was significantly higher in group III $\quad(305 \mu \mathrm{m})$ than group II (294 $\mu \mathrm{m})$ and $\mathrm{I}(286 \mu \mathrm{m})$.

$>$ Temporal, inferior, and nasal quadrants showed non-significant differences. (P-values were 0.218, 0.198 , and 0.159 , respectively). Table (3) \& figure (1).

\subsection{Perifoveal thickness}

$>$ Regarding perifoveal thickness, the temporal quadrant showed an overall significant difference between the study groups (P-value <0.001). Post hoc analysis showed that it was significantly higher in group III $(273 \mu \mathrm{m})$ than groups II $(242$ $\mu \mathrm{m})$ and I (232 $\mu \mathrm{m})$.

$>$ The nasal quadrant showed an overall significant difference between the study groups ( $\mathrm{P}$-value $<0.001)$. Post hoc analysis showed that it was significantly higher in group III $(292 \mu \mathrm{m})$ than groups II $(272 \mu \mathrm{m})$ and I $(261 \mu \mathrm{m})$.

$>$ The superior quadrant showed an overall significant difference between the study groups (P-value <0.001). Post hoc analysis showed that it was significantly higher in group III $(276 \mu \mathrm{m})$ than groups II $(252 \mu \mathrm{m})$ and I $(246 \mu \mathrm{m})$.

$>$ No significant difference was observed between the study groups regarding the inferior quadrant $(\mathrm{P}$-value $=0.059)$. Table $(4) \&$ figure $(2)$.

Table (2) Central macular thickness in the studied groups.

\begin{tabular}{|c|c|c|c|c|c|}
\hline & & $\begin{array}{l}\text { Group I } \\
(n=15 \text { eyes })\end{array}$ & $\begin{array}{l}\text { Group II } \\
(n=15 \text { eyes })\end{array}$ & $\begin{array}{l}\text { Group III } \\
(n=15 \text { eyes })\end{array}$ & P-value \\
\hline CMT & Mean \pm SD & $235 \pm 18$ & $218 \pm 27$ & $222 \pm 21$ & 0.115 \\
\hline
\end{tabular}

One-way ANOVA test was used

Table (3) Parafoveal thickness in the studied groups.

\begin{tabular}{llcccc}
\hline & Group I & Group II & Group III & \\
$(\mathbf{n = 1 5}$ eyes $)$ & $(\mathbf{n = 1 5}$ eyes $)$ & $(\mathbf{n = 1 5}$ eyes $)$ & P-value \\
\hline Temporal & Mean \pm SD & $276 \pm 24$ & $282 \pm 16$ & $287 \pm 13$ & 0.218 \\
Inferior & Mean \pm SD & $285 \pm 16$ & $290 \pm 16$ & $297 \pm 20$ & 0.198 \\
Nasal & Mean \pm SD & $289 \pm 20$ & $294 \pm 14$ & $301 \pm 16$ & 0.159 \\
Superior & Mean \pm SD & $286 \pm 21^{\mathrm{a}}$ & $294 \pm 16^{\mathrm{a}, \mathrm{b}}$ & $305 \pm 13^{\mathrm{b}}$ & 0.013 \\
\hline
\end{tabular}

One-way ANOVA test was used. Post hoc was done using Bonferroni. Different letters indicate significant pair

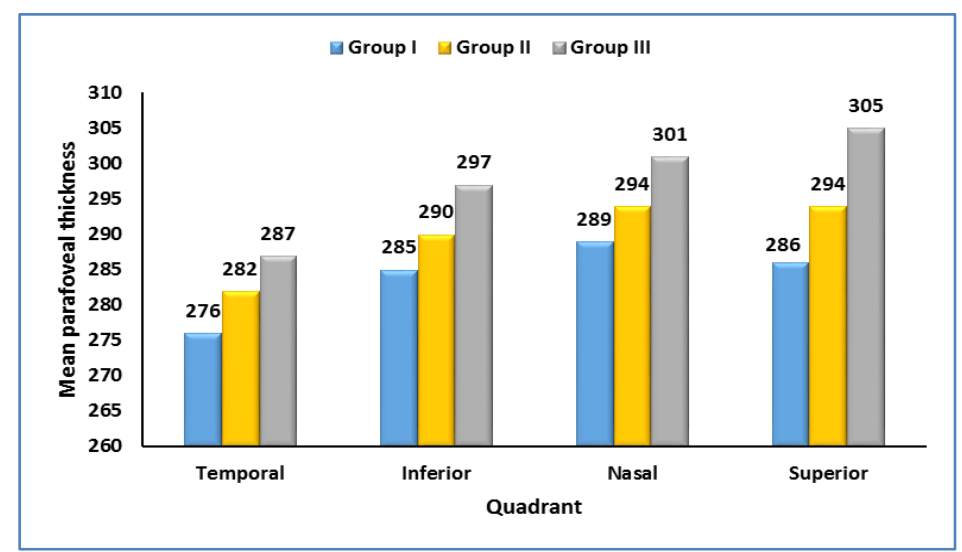

Fig. (3) Parafoveal thickness in the studied groups.

Table (4) Perifoveal thickness in the studied groups.

\begin{tabular}{llllll}
\hline & & $\begin{array}{l}\text { Group I } \\
(\mathbf{n = 1 5} \text { eyes })\end{array}$ & $\begin{array}{l}\text { Group II } \\
(\mathbf{n}=\mathbf{1 5} \text { eyes })\end{array}$ & $\begin{array}{l}\text { Group III } \\
(\mathbf{n = 1 5} \text { eyes })\end{array}$ & P-value \\
\hline Temporal & Mean \pm SD & $232 \pm 20^{\mathrm{a}}$ & $242 \pm 16^{\mathrm{a}}$ & $273 \pm 16^{\mathrm{b}}$ & $<0.001$ \\
Inferior & Mean \pm SD & $249 \pm 25$ & $262 \pm 27$ & $272 \pm 25$ & 0.059 \\
Nasal & Mean \pm SD & $261 \pm 22^{\mathrm{a}}$ & $272 \pm 14^{\mathrm{a}}$ & $292 \pm 15^{\mathrm{b}}$ & $<0.001$ \\
Superior & Mean \pm SD & $246 \pm 15^{\mathrm{a}}$ & $252 \pm 11^{\mathrm{a}}$ & $276 \pm 21^{\mathrm{b}}$ & $<0.001$ \\
\hline
\end{tabular}

One-way ANOVA test was used. Post hoc was done using Bonferroni. Different letters indicate significant pair 


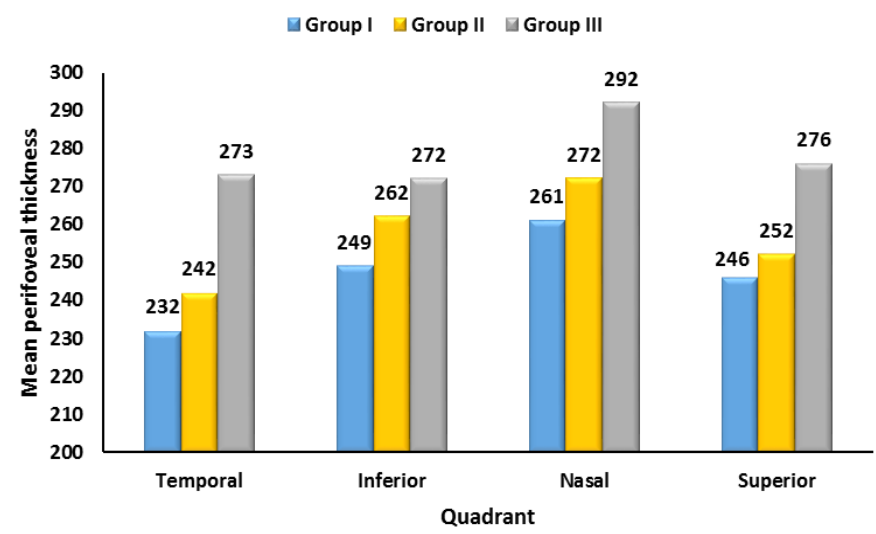

Fig. (4) Perifoveal thickness in the studied groups.

\section{Peripapillary-RNFL thickness}

Regarding Peripapillary-RNFL thickness, the inferior quadrant showed an overall significant difference between the study groups (P-value $<0.001)$. Post hoc analysis showed that it was significantly higher in group III $(149 \mu \mathrm{m})$ than groups II $(130 \mu \mathrm{m})$ and I $(120 \mu \mathrm{m})$.

$>$ The superior quadrant showed an overall significant difference between the study groups $(\mathrm{P}$-value $=0.002)$. Post hoc analysis showed that it was significantly higher in group III $(138 \mu \mathrm{m})$ than groups II $(122 \mu \mathrm{m})$ and I $(107 \mu \mathrm{m})$.

Average thickness showed an overall significant difference between the study groups ( $\mathrm{P}$-value $<0.001)$. Post hoc analysis showed that it was significantly higher in group III $(116 \mu \mathrm{m})$ than groups II $(102 \mu \mathrm{m})$ and I $(96 \mu \mathrm{m})$.

$>$ No significant difference was reported between the study groups regarding temporal and nasal quadrants. P-values were 0.341 and 0.230 , respectively Table (5) \& figure (3).

Table (5) Peripapillary-RNFL thickness in the studied groups

\begin{tabular}{llllll}
\hline & & $\begin{array}{l}\text { Group I } \\
(\mathbf{n}=\mathbf{1 5} \text { eyes })\end{array}$ & $\begin{array}{l}\text { Group II } \\
(\mathbf{n}=\mathbf{1 5} \text { eyes })\end{array}$ & $\begin{array}{l}\text { Group III } \\
(\mathbf{n}=15 \text { eyes })\end{array}$ & P-value \\
\hline Temporal & Mean \pm SD & $77 \pm 20$ & $73 \pm 8$ & $83 \pm 24$ & 0.341 \\
Inferior & Mean \pm SD & $120 \pm 18^{\mathrm{a}}$ & $130 \pm 21^{\mathrm{a}}$ & $149 \pm 14^{\mathrm{b}}$ & $<0.001$ \\
Nasal & Mean \pm SD & $78 \pm 24$ & $83 \pm 17$ & $92 \pm 24$ & 0.230 \\
Superior & Mean \pm SD & $107 \pm 23^{\mathrm{a}}$ & $122 \pm 19^{\mathrm{a}, \mathrm{b}}$ & $138 \pm 26^{\mathrm{b}}$ & 0.002 \\
Average & Mean \pm SD & $96 \pm 12^{\mathrm{a}}$ & $102 \pm 12^{\mathrm{a}}$ & $116 \pm 12^{\mathrm{b}}$ & $<0.001$ \\
\hline
\end{tabular}

One-way ANOVA test was used. Post hoc was done using Bonferroni. Different letters indicate significant pair

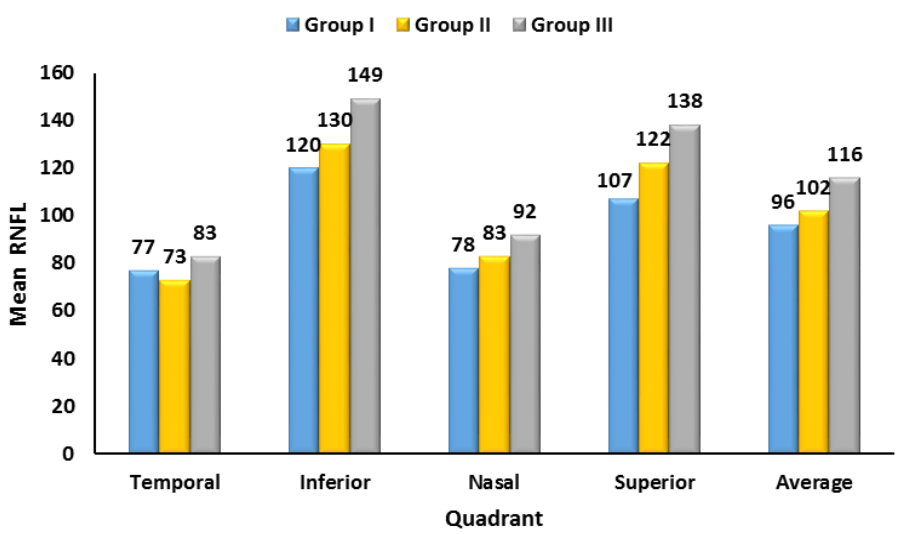

Fig. (3) Peripapillary-RNFL thickness in the studied groups. 


\begin{tabular}{|c|c|}
\hline $\begin{array}{l}\text { PATIENT } \ldots \ldots \ldots \ldots \\
\text { ID } \quad \ldots \ldots \ldots \ldots \ldots \\
\text { EYE EXAMIINED } \ldots \ldots\end{array}$ & $\begin{array}{l}\text { AMAL LABIB } \\
\text { OO, IOMHZ } \\
\text { CATARACT }\end{array}$ \\
\hline IOL CALCOLLATIONS: & REGGRESS NII \\
\hline $\begin{array}{l}K 1=0 \\
K 2=0\end{array}$ & $\left\{\begin{array}{l}\text { AXL }=25.43 \mathrm{MM} \\
\text { ACD }=3.4 \mathrm{MMM} \\
\text { LENS }=4.12 \mathrm{MM}\end{array}\right.$ \\
\hline
\end{tabular}

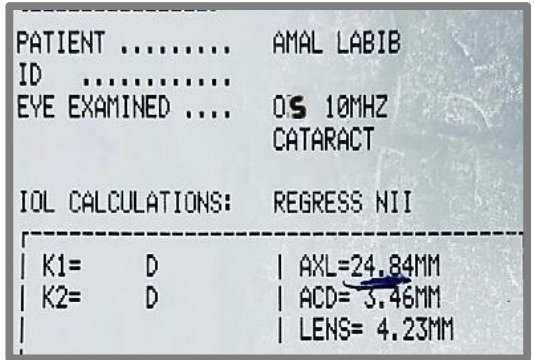

(A)

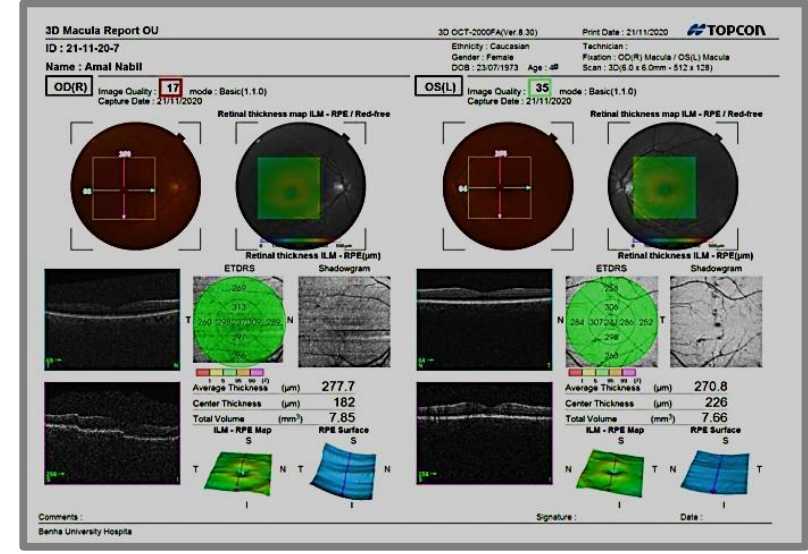

(B)

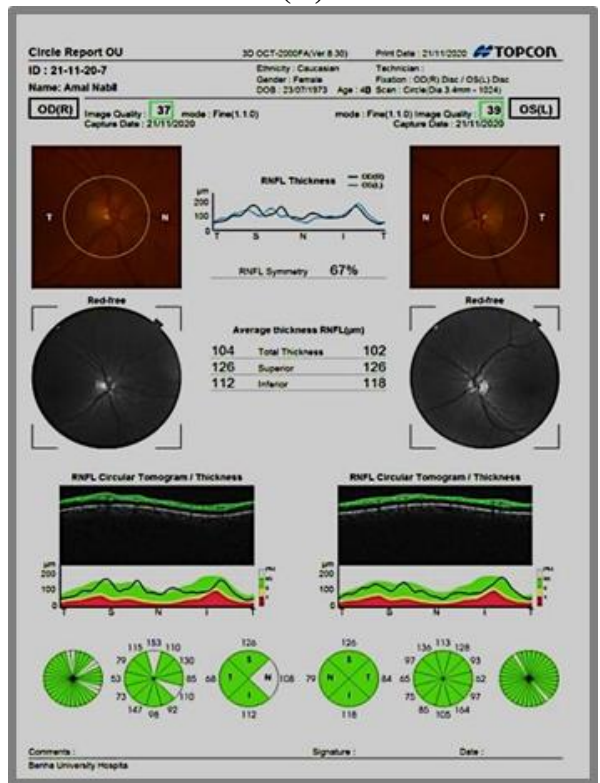

(C)

Fig. (4) Myopic case included in the study.

\section{Discussion}

Previous research has shown that as people age, their pRNFL and retinal thicknesses decline. As the number of optic nerve axons in the eye diminishes with age, age-related loss of RNFL is inevitable.

After correcting for other possible confounding variables, Mansoori and Balakrishna [10] observed that the mean RNFL thickness decreased by roughly $0.54 \mathrm{~m}$ per year as people aged throughout a threeyear follow-up period.

A regression slope of $0.53,0.44 \mathrm{~m}$ per year was established by Alamouti and Funk [11] for total retinal thickness and RNFL thickness as age increased.
RNFL thickness was assessed by Leung et al. [12] to be decreasing at a rate of $0.52 \mathrm{~m} /$ year on average, with the majority of the loss occurring in the superior $(1.35 \mathrm{~m} /$ year) and inferior (1.25\%/year) quadrants.

Poon \& et al. [13] showed that worldwide RNFL thickness decreased at a rate of $-2.0 \mathrm{~m}$ per decade, with the most influence on RNFL thickness increasing with increasing age in the superior and inferior quadrants.

According to Budenz and colleagues [6], who examined 328 healthy adults between the ages of 18 and 85 , the mean RNFL thickness dropped by around $-2 \mathrm{~m}$ for every decade older? 
In 187 normal Asian Indian eyes aged 5-75 years, Parikh and et al. [14] found a statistically significant linear reduction in RNFL thickness of roughly $0.16 \mathrm{~m}$ per year, with a plateau after age 50 .

RNFL thickness dropped by $2.71 \mathrm{~m}$ with each additional decade of life expectancy, according to Peng et al. [15]. There was higher RNFL loss as people aged beyond the age of 41 .

The present research was intended to cover participants aged 14-40 in order to minimise any negative effects of age-related retinal changes while also keeping the focus on the axial length issue. As a result, there were no age-related variations in macular or pRNFL thickness $(\mathrm{P}=0.3760)$.

A research by Rao and colleagues [16] found that patients over the age of 18 had macular measures drop by roughly 3.4 micrometres each decade, similar to our findings. The RNFL measures in Indian eyes were unaffected.

Other demographic characteristics, such as gender and eye preference, had no significant impact on macular and RNFL thickness (P-values of 0.929 and 0.871 , respectively). Because of this, these aspects aren't relevant when trying to determine what RNFL readings are typical.

Several research have confirmed our findings, including those of Hashmani and Hashmani [17], who found no association between gender and retinal or RNFL thickness. Rao and coworkers [16] discovered that gender had no impact on macular or pRNFL measures.

Our findings contradict those of Invernizzi and colleagues [18], who showed that in a healthy white population, whole retinal thickness was $15 \mathrm{~m}$ larger in men than in females over the entire posterior pole because males had thicker INL and ONL. Lam \& et al. [19] observed that the average foveal $(1 \mathrm{~mm})$ and average inner macular (1-3 $\mathrm{mm})$ thicknesses were considerably smaller in women, which is also incongruous with our findings. A smaller sample size in the present research might explain the discrepancy in findings. We encourage further investigation on the link between the two.

When we looked at the axial length influence on macular thickness in the nine ETDRS macular areas in our research, we discovered that the central macular thickness (CMT) did not vary significantly across the study groups.

Though there were no significant variations in parafoveal thickness across research groups (P-values were $0.218,0.198$, and 0.159 for the three quadrants of the eye: temporal, inferior, and nasal), there was a significant negative association between them ( $\mathrm{P}$ value $=0.013$ ) in the superior quadrant. Eyes that are hyperopic (group III) are more likely to have a refractive error of more than 13 diopters, whereas myopia (group I) is less likely.

Perifoveal thickness exhibited a strong negative connection in the superior, temporal and nasal quadrants (P-value 0.001), as thickness decreases with increasing axial length. In the lower quadrant, there was no significant difference between the research groups $(\mathrm{P}=0.059)$. We also found that in all four quadrants, the parafoveal area was substantially thicker than the perifoveal.

According to Lam and colleagues' findings, mechanical stretching of a comparable volume of retina across a wider region causes thinning of the retina. Our findings support this concept [19].

A reduction in peripheral retinal thickness may compensate for the stretching strain throughout the whole retina, preserving the central retinal thickness, which is more critical to vision if there are no significant blood vessels or optic fibres in periphery. [20].

Several results are at odds with the present research, which show that myopia increases the thickness of the fovea. With longer axial lengths, traction of the vitreous leads in elevation of the fovea, which in turn thickens it, according to Sato and colleagues [21]. This might explain why people with high myopia who are older are more likely to develop retinal diseases. According to Rao and colleagues (16), inadequate fixation with high myopia as fixation not via the fovea results in inflated foveal thicknesses. CMT has been shown to be positively associated with hyperopia in an Australian paediatric population, according to a study by Huynh and colleagues [22]. Racial and age disparities account for the majority of these variances.

Axial length was not shown to be associated with CMT in our research or others like Yau and et al. One explanation for the discrepancies is that various research employed varied sample sizes and scanning protocols on their OCT equipment.

Rao and et al. [16] found that macular thickness was negatively correlated with the eyeball's axial length in all three subfields, with the strongest negative connection seen in the superior parafoveal area. Our findings support their findings.

axial length was shown to be negatively associated with complete retinal thickness in the ETDRS outer ring, but not in the middle or foveal rings, as previously observed by Invernizzi and et al. [18].

Macular thickness reduced more in myopia in the 3-6 $\mathrm{mm}$ outer ring than at the $1-3 \mathrm{~mm}$ inner ring, according to Lam and colleagues [19].

According to Liu et al. [24], when axial length increases, the macular thickness decreases, notably in the periphery of the retina. Myopia had no effect on the average thickness of the macular retina in the middle.

The para- and perifoveal-thicknesses of the macula were shown to be adversely linked with AL by Zhao et al. Foveal thickness, on the other hand, was not linked to ALS.

In contrast to what we found in the research, For myopes, Lim et al. [20] observed that axial length was related to both thinner parafovea and thicker fovea.

It was also discovered by $\mathrm{Wu}$ et al. [26] that the foveola and focal area of the retina are thicker in those with severe myopia, whereas the inner and outer macular regions are much thinner.

Also, according to Chung et al. [27] the fovea was thicker, the parafovea and perifovea were narrower, 
and the axial length started at $25.5-26.0 \mathrm{~mm}$ the slope was steeper.

In young adults, Lee et al. [28] found no connection between axial length and other measures of retinal thickness in any macular areas.

As a result of this study, we found that the average nasal RNFL thickness was greater than the superior nasal RNFL thickness ( $\mathrm{I}=10723 \mathrm{~m}, \mathrm{II}=122$ 19m, $\mathrm{III}=138.26 \mathrm{~m})$, followed by the temporal quadrant $(\mathrm{I}=77.20)$ and the temporal nasal quadrant $(\mathrm{I}=73.8 \mathrm{~m})$. The "double hump pattern" was evident in our findings, as was the "ISNT rule," which indicates that the inferior neuroretinal rim is the widest followed by the superior, nasal, and temporal neuroretinal rims, and that this was true in all three eye conditions: myopia, emmetropia, and hyperopia.

In a study of 100 eyes of high myopes, Rao et al. [16] observed that the temporal RNFL was narrower than the nasal RNFL. These findings are corroborated by histological evidence.

138 healthy myopic participants were involved in a cross-sectional observational research by Qiu et al. [29] and the ISNT rule was shown to be intact in $63 \%$ of them.

Malakar et al. [30] discovered that in both myopes and emmetropes, the RNFL thickness had a distinctive "double hump pattern" and was governed by the "ISNT rule.

According to Chan et al. [31] only $15.7 \%$ of nonglaucomatous eyes followed the ISNT rule in the rim region, indicating substantial poor compliance with the ISNT rule.

In another research, just $18 \%$ of normal eyes exhibited rim regions that followed the ISNT rule in 280 Caucasian patients, according to Nayak et al.

Because the RNFL thickness was much larger in the hypermetropic group (III) than the emmetropic or myopic groups in our research, we concluded that it had a negative connection with axial length (II and I, respectively). In both the superior and inferior quadrants, this was statistically significant. In terms of the temporal and nasal quadrants, however, there was no significant difference seen between the research groups (P-values $=0.341$ and 0.230 , respectively).

RNFL axon number is proportional to $\mathrm{AL}$, but $\mathrm{AL}$ size generates a bigger surface area, and the dispersion of an equal number of retinal nerves throughout the larger surface area causes a thinner layer. RNFL thickness and AL were shown to be negatively correlated by Parvaresh et al. [33] in all four quadrants.

The RNFL thickness was shown to be negatively correlated with the axial length of the eyeball in accordance with us by Rao et al. [16] in the superior, nasal, and inferior quadrants.

To their surprise, Dikkaya and Erdur [34] found that high hyperopes exhibited thicker RNFL than low hyperopes and that RNFL correlated negatively with axial length in children.

Mehboob et al. [35] found a substantial negative connection between axial length and the mean pRNFL thickness in each quadrant.
Using emmetropic eyes, Oner and colleagues [36] showed that the thickness of the pRNFL is thinner in the superior, inferior, and temporal quadrants in myopic eyes, whereas the thickness is larger in the nasal quarter in hyperopic eyes.

To summarise, Hoffmann et al. [37] found that an increased axial length was related with a smaller plasma RNFL, with the average thickness of the pRNFL decreasing by $1.66 \mathrm{~m}$ for every extra millimetre of axial length.

OCT measurements of AL and average RNFL thickness showed a negative connection, according to Peng et al. [15]. The RNFL was $1.78 \mathrm{~m}$ narrower for every $\mathrm{mm}$ increase in AL, with the inferior quadrant showing the largest trend for RNFL loss as $\mathrm{AL}$ increased $(4.46 \mathrm{~m} / \mathrm{mm})$

Axial length was shown to be significantly associated with RNFL thickness by Rauscher et al. Myopia-related RNFL was shown to be thinner in the superior and inferior portions of myopic patients, with an overall drop in RNFL thickness of $7 \mathrm{~m}$ for every $1 \mathrm{~mm}$, a figure larger than that previously recorded for other populations.

According to Budenz et al. [6], those with longer eyes or eyes that are more myopic had RNFLs that were $2 \mathrm{~m} / \mathrm{mm}$ thinner. According to Leung and coworkers [39], the velocity was $2.75 \mathrm{~m} / \mathrm{mm}$, but Salchow et al.

Prior research like Hoh and et al. [41, 42] and Melo and et al. [41, 42] found no link between myopia and RNFL thickness, however this one did. Early OCT and confocal laser systems had lesser sensitivity and inferior resolution, therefore these research may have been constrained.

According to Invernizzi et al., [18], there was no correlation between RNFL thickness and any of the three factors of age, gender, or AL. This discrepancy was attributed to the geographic location of the study. The thickness of the retinal nerve fibre layer (RNFL) is often measured via a circumpapillary scan, which includes fibres from all of the retina. The research, on the other hand, only looked at a section of the retinal nerve fibres by measuring the thickness of the layer over the macula.

There was no significance discovered by Lim et al., [20], who included a broader range of myopes in their investigation.

Both hyperopes and myopes were included in the study by Song et al. [43] and no significant link was discovered between AL and RNFL thickness.

\section{Conclusion}

It was found that the lengthening of the AL has an inverse relationship with macula retinal thickness in the superior, nasal, and temporal quadrants as well as on inner parafovea in the superior quadrant with no corresponding relationship on the centre macula when three groups were compared: myopes, emmetropes, and hypermetropes. In all three groups, pRNFL exhibited the distinctive "double hump pattern" and adhered to the "ISNT rule." When it came to the temporal and nasal quadrants, there was no statistically significant difference between the 
research groups in terms of RNFL thickness and axial length. To accurately assess the thickness of various retinal tissues, such as RNFL, OCT is a helpful tool. This helps in early glaucoma diagnosis, follow-up, and treatment of patients to prevent them from irreversible vision loss.

\section{References}

[1] M.Sugita, M.Pircher, S.Zotter, B.Baumann, P.Roberts, T.Makihira, N.Tomatsu, M.Sato, C.Vass and C. K. Hitzenberge, Retinal Nerve Fiber Bundle Tracing and Analysis in Human Eye by Polarization Sensitive OCT . Biomedical OpticsExpress.vol.6,pp.1030-1054,2015.

[2] D.C.Hood, AS.Raza, C.G.de Moraes, V.Liebmann and R.J.M.Ritch, Glaucomatous Damage of the Macula. Progress in Retinal and Eye Research. vol.32, pp.1-21. 2013.

[3] V.Sowmya, V. R.Venkataramanan and V.Prasad, Effect of Refractive Status and Axial Length on Peripapillary Retinal Nerve Fiber Layer Thickness: An Analysis Using 3D OCT. J Clin Diagn Res. vol. 9, pp. 1-4,2015.

[4] S. E.Yanni, J.Wang, C. S.Cheng, K. I.Locke, Y.Wen, D. G.Birch and E. E.Birch, Normative Reference Ranges for The Retinal Nerve Fiber Layer, Macula, and Retinal Layer Thicknesses in children. American Journal of Ophthalmology. vol. 155 , pp. 354-360,2013.

[5] V.Oner, V.Aykut, M.Tas, M. F.Alakus, and Y. Iscan, Effect of Refractive Status on Peripapillary Retinal Nerve Fiber Layer Thickness: A Study by RTVue Spectral Domain Optical Coherence Tomography. British Journal of Ophthalmology. vol. 97, pp. 75-79,2013.

[6] D. L.Budenz, D.R.Anderson, R.Varma, J.Schuman, L.Cantor, J. Savell, D. S.Greenfield, V. M. Patella, H. A. OD. Quigley and J.Tielsch, Determinants of Normal Retinal Nerve Fiber Layer Thickness Measured by Stratus OCT. Ophthalmology. vol. 114, pp. 1046-1052, 2007.

[7] K.Ueda, A.Kanamori, A.Akashi, M.Tomioka, Y.Kawaka, and M.Nakamura, Effects of Axial Length and Age on Circum papillary Retinal Nerve Fiber Layer and Inner Macular Parameters Measured by 3 Types of SD-OCT Instruments. Journal of Glaucoma.vol. 25, pp. 383-389,2016.

[8] M. S.Kolega, S.Kovacevic, S.Canovic, A. D.Pavicic, and J.K.Basic, Comparison of IOLMaster and Ultrasound Biometry in Preoperative Intra Ocular Lens IOL Power Calculation. Coll Antropol.vol. 39, pp. 233-235. 2015.

[9] K. J.Hoffer, Ultrasound Velocities for Axial Eye Length Measurement. Journal of Cataract \& Refractive Surgery.vol.20, pp. 554-562. 1994.

[10] T.Mansoori and N.Balakrishna, Effect of Aging on Retinal Nerve Fiber Layer Thickness in Normal Asian Indian Eyes: A Longitudinal Study. Ophthalmic Epidemiology.vol. 24, pp. 24-28, 2016.

[11] B.Alamouti, J.Funk, Retinal Thickness Decreases with Age: an OCT study. $\mathrm{Br} \mathrm{J}$ Ophthalmol.vol. 87, pp. 899-901, 2003.
[12] C. K.S.Leung, M.Yu, R. N.Weinreb, C. Ye, S.Liu, G.Lai, and D.S.C.Lam, Retinal Nerve Fiber Layer Imaging with Spectral-Domain Optical Coherence Tomography. Ophthalmology.vol.119, pp. 731-737, 2012.

[13] L.Y.C.Poon, H.Antar, E.Tsikata, R.Guo, G.Papadogeorgou, M.Freeman, Z.Khoueir, R.Lee, E.Shieh, H.Simavli, C. J.Que, J. F. deBoerand, T. C.Chen, Effects of Age, Race, and Ethnicity on the Optic Nerve and Peripapillary Region using Spectral-Domain OCT 3D Volume Scans. Trans Vis Sci Tech.vol. 7, pp. 12, 2018.

[14] R. S.Parikh, S. R.Parikh, G. C.Sekhar, S.Prabakaran, J. G.Babu, and R.Thomas, Normal Age-Related Decay of Retinal Nerve Fiber Layer Thickness. Ophthalmology.vol.114,pp.921926,2007.

[15] P. H.Peng, S. Y.Hsu, W. S.Wang, and M.L.Ko, Age and axial length on Peripapillary Retinal Nerve Fiber Layer Thickness Measured by Optical Coherence Tomography in NonGlaucomatous Taiwanese participants. Plos One.vol.12, pp. e0179320,2017.

[16] Rao, H. L., Kumar, A. U., Babu, J. G., Kumar, A., Senthil, S., and Garudadri, C. S. Predictors of Normal Optic Nerve Head, Retinal Nerve Fiber Layer, and Macular Parameters Measured by Spectral Domain Optical Coherence Tomography. Investigative Opthalmology \& Visual Science: vol. 52, no. 2, pp. 1103-1110. 2011.

[17] Hashmani, N., and Hashmani, S. Threedimensional mapping of peripapillary Retinal Layers Using A Spectral Domain Optical Coherence Tomography. Clinical Ophthalmology: vol. 11, pp. 2191-2198. 2017.

[18] Invernizzi, A., Pellegrini, M., Acquistapace, A., Benatti, E., Erba, S., Cozzi, M., Cigada, M.,Viola, F., Gillies, M., and Staurenghi, G. Normative Data for Retinal-Layer Thickness Maps Generated by Spectral-Domain OCT in a White Population. Ophthalmology Retina: vol. 2, no. 8, pp. 808-815. 2018.

[19] Lam, D. S. C., Leung, K. S., Mohamed, S., Chan, W., Palanivelu, M. S., Cheung, S. Y. L., Li, E. Y. M., Lai, R. Y. K., and Leung, C. K. Regional Variations in The Relationship between Macular Thickness Measurements and Myopia. Invest. Ophthalmol. Vis. Sci: vol. 48, pp. 376382. 2007.

[20] Lim, M. C. C., Hoh, S.-T., Foster, P. J., Lim, T.-H., Chew, S.-J., Seah, S. K. L., and Aung, T. Use of Optical Coherence Tomography to Assess Variations in Macular Retinal Thickness in Myopia. Investigative Opthalmology \& Visual Science: vol. 46, no, 3, pp. 974- 978. 2005.

[21] Sato, A., Fukui, E., and Ohta, K. Retinal Thickness of Myopic Eyes Determined by Spectralis Optical Coherence Tomography. British Journal of Ophthalmology: vol. 94, no, 12, pp. 1624-1628. 2010. 
[22] Huynh, S. C., Wang, X. Y., Rochtchina, E., and Mitchell, P. Distribution of Macular Thickness by Optical Coherence Tomography: Findings from a Population-Based Study of 6Year-Old Children. Investigative Opthalmology \& Visual Science: vol. 47, no. 6, pp. 2351- 2357. 2006.

[23] Yau, G. S. K., Lee, J. W. Y., Woo, T. T. Y., Wong, R. L. M., and Wong, I. Y. H. Central Macular Thickness in Children with Myopia, Emmetropia, and Hyperopia: An Optical Coherence Tomography Study. BioMed Research International: 2015, 1-4. 2015.

[24] L.Liu, J.Zou, L.Jia, J.Yang, and S.Chen, Spectral- and Time-Domain Optical Coherence Tomography Measurements of Macular Thickness in Young Myopic Eyes. Diagnostic Pathology. vol. 9, pp. 38,2014.

[25] Z.Zhao, X.Zhou, C.Jiang, and X.Sun, Effects of Myopia on Different Areas and Layers of the Macula: a Fourier-Domain Optical Coherence Tomography Study of a Chinese Cohort. BMC Ophthalmology.vol. 15, pp. 90, 2015.

[26] P.C.Wu, Y.J.Chen, C.H.Chen, Y.H.Chen, S.J.Shin, H.J.Yang, and H.K. Kuo, Assessment of Macular Retinal Thickness and Volume in Normal Eyes and Highly Myopic Eyes with Third-Generation Optical Coherence Tomography.Eye.vol.22,pp.551-555,2007.

[27] Y. W.Chung, M. Y.Choi, J.S.Kim, and J.W.Kwon, The Association between Macular Thickness and Axial Length in Myopic Eyes. Biomed Res Int 8913582, 2019.

[28] S.S.Y.Lee, G.Lingham, D.Alonso-Caneiro, J.Charng, F. K.Chen, S.Yazar, and D.A.Mackey, Macular Thickness Profile and Its Association With Best-Corrected Visual Acuity in Healthy Young Adults. Trans Vis Sci Tech.vol. 10, pp. 8, 2021.

[29] K.Qiu, G.Wang, X.Lu, R.Zhang, L.Sun, and M. Zhang, Application of The ISNT Rules on Retinal Nerve Fiber Layer Thickness and Neurortinal Rim Area in Healthy Myopic Eyes. Acta Ophthalmologica.vol.96,pp.161-167, 2017.

[30] M.Malakar, S. N.Askari, H.Ashraf, A.Waris, A.Ahuja, and A.Asghar, Optical Coherence Tomography Assisted Retinal Nerve Fiber Layer Thickness Profile in High Myopia. Journal Of Clinical And Diagnostic Research.vol. 9, pp. NC01-NC03, 2015.

[31] E. W.Chan, J.Liao, R.Chao Ming Foo, S. C.Loon, T.Aung, T. Y.Wong, and C.Y. Cheng, Diagnostic Performance of the ISNT Rule for Glaucoma Based on the Heidelberg Retinal Tomograph. Translational Vision Science \& Technology.vol. 2,pp. 2, 2013.

[32] N.V.Nayak, T.L.Berezina, R.D. Fechtner, M.J.Sinai and A.S.Khouri, Effect of Age and Disc Size on Rim Order Rules by Heidelberg Retina Tomograph. Journal of Glaucoma.vol.24,pp.377-382, 2015.

[33] M.M.Parvaresh, M.Imani, M.BahmaniKashkouli and M.Soltan-Sanjari, ptical
Coherence Tomography-Measured Nerve Fiber Layer and Macular Thickness in Emmetropic, High-Myopic and High-Hyperopic Eyes. Iranian Journal of Ophthalmology: vol. 20, no. 2, pp.4-9. 2008.

[34] F.Dikkaya and S. K.Erdur, Comparison of Optical Coherence Tomography Measurements between High Hyperopic and Low Hyperopic Children. Therapeutic Advances in Ophthalmology.vol.12, pp. 1- 10,2020.

[35] M.A.Mehboob,Q.U.Islam and M.A.Yaqub, Effect of Axial Length on Peripapillary Retinal Nerve Fiber Layer Thickness Measured by Spectral Domain Optical Coherence Tomography. Pak Armed Forces Med J: vol. 67, pp.238-242. 2017.

[36] Oner, V., Taş, M., Türkcü, F. M., Alakuş, M. F., Işcan, Y., and Yazıcı, A. T. Evaluation of Peripapillary Retinal Nerve Fiber Layer Thickness of Myopic and Hyperopic Patients: A Controlled Study by Stratus Optical Coherence Tomography. Current Eye Research: vol. 38, no. 1, pp.102-107. 2012.

[37] Hoffmann, E. M., Schmidtmann, I., Siouli, A., Schuster, A. K., Beutel, M. E., Pfeiffer, N., and Lamparter, J. The Distribution of Retinal Nerve Fiber Layer Thickness and Associations with Age, Refraction and Axial Length: the Gutenberg health study. Graefe's Archive for Clinical and Experimental Ophthalmology: vol. 256, no9, pp.1685-1693. 2018.

[38] Rauscher, F. M., Sekhon, N., Feuer, W. J., and Budenz, D. L. Myopia Affects Retinal Nerve Fiber Layer Measurements as Determined by Optical Coherence Tomography. Journal of Glaucoma: vol. 18, no.7, pp.501-505. 2009.

[39] C. K.S.Leung, S.Mohamed, Leung, K. S., Cheung, C. Y.-L., Chan, S. L., Cheng, D. K., Lee, A. K., Leung, G. Y., Rao, S. K., and Lam, D. S. C. Retinal Nerve Fiber Layer Measurements in Myopia: An Optical Coherence Tomography Study. Investigative Opthalmology \& Visual Science: vol. 47, no. 12, pp.5171- 5176, 2006.

[40] D.J.Salchow, Y.S.Oleynikov, M.F.Chiang, S.E.Kennedy-Salchow, K.Langton, J.C.Tsai, and L.A.Al-Aswad, Retinal Nerve Fiber Layer Thickness in Normal Children Measured with Optical Coherence Tomography. Ophthalmology.vol.113,pp.786-791,2006.

[41] S.T.Hoh, M.C.C.Lim, S.K.L.Seah, T.H.Lim, S.J.Chew, P.J.Foster, Aung, Peripapillary Retinal Nerve Fiber Layer Thickness Variations with Myopia. Ophthalmology, vol.5, pp. 773-777, 2006.

[42] G.B.Melo, R.D.Libera, A.S.Barbosa, L.M.G.Pereira, L.M.Doi and .A.S.Melo, Comparison of Optic Disk and Retinal Nerve Fiber Layer Thickness in Nonglaucomatous and Glaucomatous Patients With High Myopia. American Journal of Ophthalmology.vol.142,pp. 858-860,2006. 
[43] W.Song, D.Ren, W.Li, L.Jiang, KW.Cho, P.Huang, C.Fan, Y.Song, Y.Liu, L.Rui, SH2B regulation of growth, metabolism, and longevity in both insects and mammals. Cell Metab.vol.115, pp.427-37, 2010. 